\title{
Study of Extraordinary Transmission in a Circular Waveguide System
}

\author{
Francisco Medina, Fellow, IEEE, Francisco Mesa, Member, IEEE, Jorge A. Ruíz-Cruz, \\ Jesús M. Rebollar, and José R. Montejo-Garai
}

\begin{abstract}
Extraordinary transmission through periodic distributions of sub-wavelength holes made in opaque screens has been demonstrated and exhaustively studied along the last decade. More recently, extraordinary transmission has also been predicted and experimentally observed through electrically small diaphragms located inside hollow pipe waveguides. This last phenomenon cannot be explained in terms of surface waves excited along the periodic system (the so-called surface plasmon polaritons). Transverse resonances can be invoked, however, as a sound explanation for extraordinary transmission in this kind of systems. In this paper, a simple waveguide system, exhibiting exactly the same behavior previously observed in periodic 2-D arrays of holes, is analyzed in depth. Analogies and differences with the periodic case are discussed. The theoretical and experimental results reported in this paper provide strong evidence in favor of the point of view emphasizing the concept of impedance matching versus surface wave excitation. The role of material losses is discussed as an important practical issue limiting the maximum achievable subwavelength transmission level. Most of our conclusions can be applied to both periodic arrays of holes and diaphragms in closed waveguides.
\end{abstract}

Index Terms-Circular waveguide discontinuities, extraordinary transmission, impedance matching.

\section{INTRODUCTION}

$\mathbf{T}$ HE electromagnetic phenomenon known as extraordinary (optical) transmission has been exhaustively studied by many physicists and electrical engineers since its discovery at

Manuscript received January 23, 2010; revised March 16, 2010; accepted March 23, 2010. Date of publication May 10, 2010; date of current version June 11, 2010. This work was supported by the Spanish Ministerio de Ciencia e Innovación and European Union FEDER funds under Projects TEC2007-65376, Project Consolider-Ingenio 2010 "Engineering Metamaterials" (Project CSD2008-00066), and by the Spanish Junta de Andalucía under Project TIC-4595. The work of F. Medina was supported by the Mobility for Human Resources Programme of the Spanish Ministry of Science and Innovation under Grant P2009-0405), which funded his stay with the Department of Electronic Engineering, Queen Mary University of London (QMUL), London, U.K.

F. Medina is with the Microwaves Group, Department of Electronics and Electromagnetism, Faculty of Physics, University of Seville, 41012-Seville, Spain (e-mail: medina@us.es).

F. Mesa is with the Microwaves Group, Department of Applied Physics 1, ETS de Ingeniería Informática, University of Seville, 41012-Seville, Spain (e-mail: mesa@us.es).

J. A. Ruiz-Cruz is with the Escuela Politécnica Superior, Universidad Autónoma de Madrid, E-28049 Madrid, Spain (e-mail: Jorge.RuizCruz@uam. es).

J. M. Rebollar and J. R. Montejo-Garai are with the Departamento Electromagnetismo y Teoría de Circuitos, Universidad Politécnica de Madrid, 28040Madrid, Spain (e-mail: jmrm@etc.upm.es; jr@etc.upm.es).

Color versions of one or more of the figures in this paper are available online at http://ieeexplore.ieee.org.

Digital Object Identifier 10.1109/TMTT.2010.2048254 the optical frequency range [1]. Authoritative in-depth reviews are available today [2]-[4]. In spite of the use of the word optical, this enhanced frequency-selective transmission can be observed at any frequency range [5]-[9]. In most situations, extraordinary transmission is linked to either the existence of a periodic (or pseudoperiodic in the case of cylindrically symmetrical systems) perturbation of the region around an individual sub-wavelength hole [10]-[13] or to the existence of a fully periodic structure whose unit cell includes the sub-wavelength hole. The phenomenon is also observed in 1-D periodic gratings involving sub-wavelength slits [14]-[16]. Though any real system is not completely periodic because of the limited size of the diffraction grid and/or the illuminating spot (see, for instance, the discussion in [17]), the fundamental physics of the phenomenon can be accurately accounted for by the study of an ideal periodic system excited by a uniform plane wave. This, in turn, can be reduced to the study of a single unit cell.

Currently, the dominant paradigm associates extraordinary transmission with the ability of periodically structured surfaces to support surface waves. These waves (named spoof plasmons) mimic the behavior of optical surface plasmon polaritons supported by metal/dielectric interfaces [18]-[22]. However, some researchers have reported extraordinary transmission through periodic structures in cases for which the polarization precludes the excitation of surface plasmon polaritons [23]. Even more relevant in the context of this paper is the prediction of the existence of extraordinary transmission at microwave/millimeter-wave frequencies through electrically small diaphragms placed inside hollow pipe waveguides [24]-[29]. Experimental evidence of this type of transmission has recently been reported by the authors in [30]. In that paper, the authors experimentally show how an off-centered diaphragm inside a circular cross-section waveguide exhibits the same extraordinary phenomenon previously studied in 2-D periodic arrays of holes. This result was theoretically predicted in [24] using a mode-matching analysis. It is apparent that surface plasmon polaritons do not play any role in the hollow pipe waveguide situation. Instead of surface waves, eigenmodes of the type discussed in [24], [29], and [31] should be considered to be responsible for this extraordinary transmission. This kind of resonances has also been considered responsible of extraordinary transmission through 2-D arrays of holes in an early paper [32]. Recently, some of the authors of this paper proposed an alternative paradigm to explain extraordinary transmission phenomena through 2-D [26] and 1-D [33] arrays of apertures. This approach leads to simple circuit-like models whose parameters can be evaluated with very small computational effort. The model lies on the 
concept of impedance matching (very familiar to microwave engineers) rather than on the concept of intermediary surface wave excitation. A first advantage of such a point of view is that it provides a unified framework to explain extraordinary transmission, not only through periodic arrays of apertures (1-D or 2-D), but also through small diaphragms placed inside closed waveguides. Another practical advantage of our proposal is that circuit models have only a few parameters to be determined. Some parameters are known in closed form (from dimensions and electrical properties), while others can easily be extracted from a few full-wave simulations. In some sense, a circuit model can be viewed as an ad hoc reduced order model, which, once the parameters are known, quickly gives the wideband response of the structure. Moreover, with some basic knowledge of circuit and waveguide theory, it is relatively simple to predict the qualitative behavior of a new structure without performing any involved computation.

In this paper, we will study in depth the transmission through subwavelength off-centered rectangular and elliptical apertures located inside a circular waveguide. Experimental and theoretical results will show that this system behaves as previously studied 2-D arrays of holes. In fact, the provided theoretical explanation for the observed transmission spectra is the same for both kind of systems. In our opinion, this analogy clearly supports the idea that the nature of the phenomenon could be more general than previously suggested, in perfect agreement with the point of view subscribed by the authors of [34], although using rather different arguments. The structure under study will also be used to analyze the significant influence of losses in this kind of systems. This issue is very important because of its practical implications when the size of the aperture is small. The effect of losses is theoretically anticipated using our conceptual scheme and then experimentally demonstrated. The influence of the thickness of the screen, and the nature of the fields around critical frequencies, is also explored and discussed.

\section{Thin Diaphragm Inside A Closed Circular WAVEGUIDE}

In this section, we will focus on the study of a particular waveguide discontinuity: an off-centered diaphragm inside a hollow circular waveguide (see Fig. 1). The choice of a circular waveguide, rather than rectangular, is mainly motivated by its close "modal" correspondence with the unit cell of a 2-D periodic array of holes. The equivalent-circuit model for the circular waveguide with a diaphragm will be shown to be almost identical to that used in [26]. In this section, we will first study the simplest case: a lossless system with very small diaphragm thickness. Later, the equivalent circuit will be slightly modified, adding a resistor, to account for losses. The qualitative theoretical predictions will be compared with experimental data, thus showing the strong impact of losses when the holes are very small. The influence of the geometry of the hole will also be briefly discussed by comparing a rectangular hole with an elliptical one. The full-wave numerical data presented in this paper have been computed by using either a homemade optimized high-performance code based on mode matching (following the guidelines in [35] and [36]) or the commercial fi-
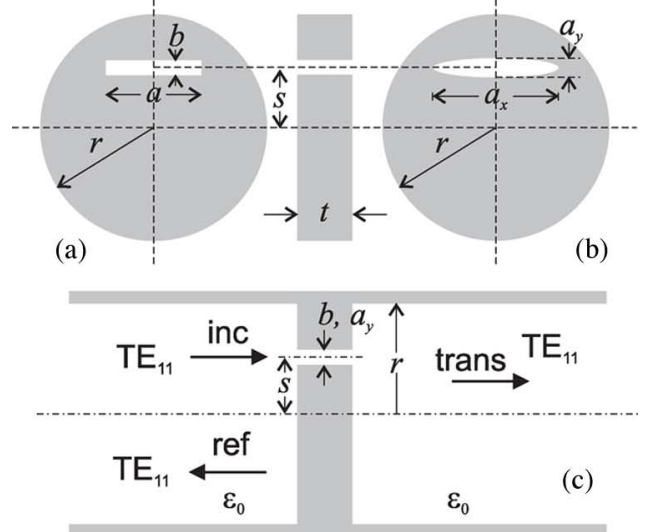

(b)

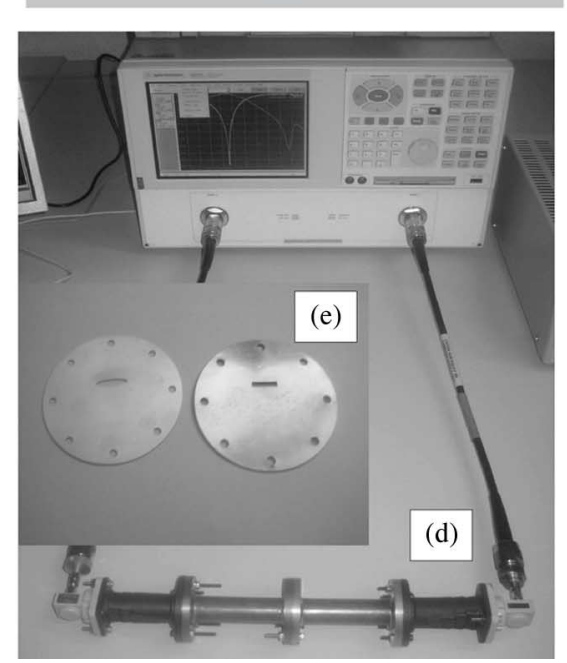

Fig. 1. (a) Rectangular and (b) elliptical diaphragms that have been placed inside a circular cross-section waveguide. Note that $s$ denotes the distance between the centers of the waveguide and the hole. In our experiments, $a=$ $10.0 \mathrm{~mm}, b=2.0 \mathrm{~mm}, s=7.0 \mathrm{~mm}$ (rectangular hole) or $a_{x}=13.0 \mathrm{~mm}$, $a_{y}=3.0 \mathrm{~mm}, s=6.0 \mathrm{~mm}$ (elliptical hole). (c) Schematics of the discontinuity problem studied in this paper using mode matching and circuit modeling. (d) Experimental setup including coax to rectangular waveguide and rectangular-to-circular waveguide transitions. (e) Detail of the two diaphragms employed.

nite-element electromagnetic solver High Frequency Structure Simulator (HFSS).

\section{A. Zero-Thickness Diaphragms: Statement of the Problem and Basic Theory}

The problem to be considered here is the possibility of total (or enhanced) transmission of electromagnetic waves through an electrically small aperture made in a thin metal plate that covers the cross section of a circular waveguide (the thick case will be treated later). Fig. 1 shows the schematics of the problem and also the two different rectangular and elliptical diaphragms used in our study. If an electromagnetic wave impinges on a single sub-wavelength hole practiced in a flat metal screen, it is clearly expected that the level of transmission through the hole is low (this is the case for the diaphragm dimensions reported in the caption to Fig. 1 and for frequencies below the cutoff of the first higher order mode of the circular waveguide, namely, $11.77 \mathrm{GHz}$ ). It is known from accepted extraordinary transmission theory [3], [4], however, that periodically grouping many of these sub-wavelength holes gives rise to high transmis- 


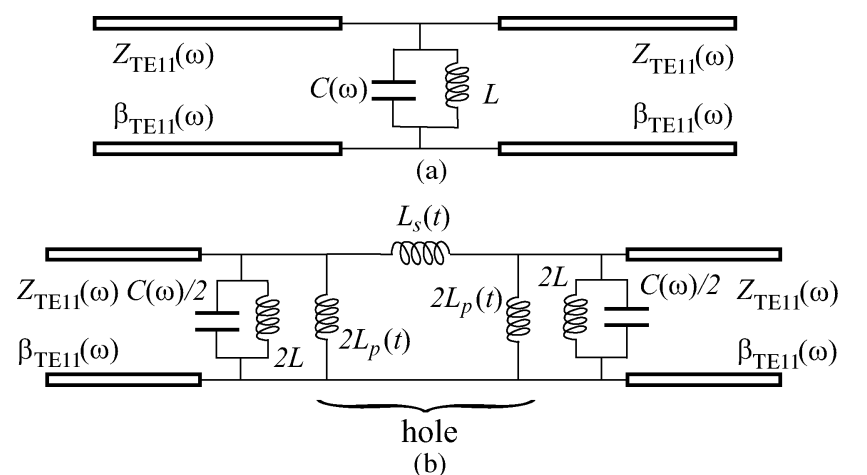

(b)

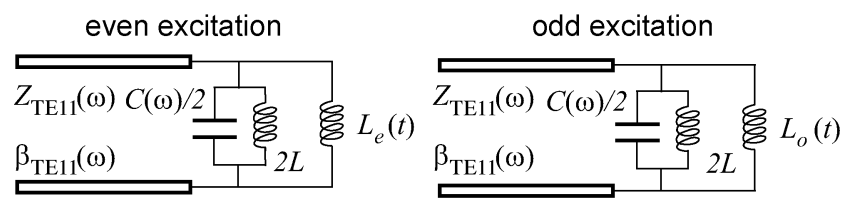

(c)

Fig. 2. (a) Equivalent circuit for very thin diaphragms in the circular waveguide ( $\omega$ stands for the angular frequency). (b) Equivalent circuit for arbitrary thickness of the perforated plate (the inductances of the $\pi$-circuit depend on the thickness $t$ of the diaphragm). (c) Equivalent circuits used for even and odd symmetry excitations. In these circuits, $L_{e}=2 L_{p}$ and $L_{o}=2 L_{p} \|\left(L_{s} / 2\right)$.

sion peaks observed at frequencies for which the wavelength is larger, but close to, the period of the structure. Our question now is if a similar effect takes place when the hole is located inside a hollow waveguide. The theory based on excitation of spoof surface plasmon polaritons cannot help us here because these waves have no meaning in this context. However, the theories reported in [26] and [24], [28], and [29] predict that extraordinary transmission should also happen in this situation. The excitation by the discontinuity of an evanescent near-cutoff TM mode of the waveguide would provide the mechanism to achieve perfect matching impedance, and thus, total transmission.

From the microwave engineer's point of view, the problem can be viewed as the textbook analysis of a diaphragm discontinuity [37], [38]. The fundamental $\mathrm{TE}_{11}$ mode supported by the circular waveguide impinges on the aperture and is scattered back and forth. In particular, we will be interested in the reflection and transmission coefficients of that mode for frequencies below the cutoff of higher order modes. In this frequency range, higher order scattered modes will store the excess of electric (TM modes) or magnetic (TE modes) energy around the discontinuity. This electromagnetic fact can be accounted for by means of appropriately chosen capacitances and inductances in an equivalent circuit model [37], [38], such as that shown in Fig. 2(a) (which remains valid for finite, but small thicknesses, such as in our case: $t=0.35 \mathrm{~mm}$ ). In this figure, the $\mathrm{TE}_{11}$ mode propagating along the uniform circular waveguide sections at both sides of the discontinuity is represented by two transmission lines. This equivalent circuit is very close to the one used in [26] for periodic 2-D arrays of holes with the only differences coming from the frequency-dependent behavior of the characteristic impedance $Z_{\mathrm{TE}_{11}}(\omega)$ and propagation constant $\beta_{\mathrm{TE}_{11}}(\omega)$ of the transmission line used now.

In the present case, we deal with a dispersive waveguide mode, while in [26], we dealt with a TEM nondispersive mode. The values of $L$ and $C$ have been easily extracted from a few frequency values of the numerically computed scattering parameters, as shown in Appendix A. In [26], it was shown that this model predicts extraordinary (total) transmission at a frequency slightly below the cutoff frequency of the first higher order TM mode supported by the relevant waveguide. The relevant waveguide (unit cell) for normal incidence in [26] was an ideal parallel-plate waveguide; the first higher order mode was $\mathrm{TM}_{02}$. The presence of the transmission peak was associated with the singular behavior of $C$ [see Fig. 2(a)] near the cutoff frequency of the $\mathrm{TM}_{02}$ mode [26, eqs. (2) and (3)]. The lack of consideration of such behavior is, likely, one of the reasons of the late discovery of the extraordinary transmission in the microwave community. In the circular waveguide problem under study, the mode providing the singular behavior of $C$ is now the $\mathrm{TM}_{01}$ mode. The symmetry of the fields of this mode (together with the symmetry of the impinging field pattern) forces the use of off-centered holes ( $s \neq 0$ in Fig. 1) to ensure its excitation. If the same rationale used in [26] is applied to the present problem, our equivalent-circuit model predicts total transmission of electromagnetic waves-provided losses are neglected-at some frequency below and near the cutoff frequency of this $\mathrm{TM}_{01}$ mode. In [30], numerical and experimental results are reported proving this fact. The experimental results in [30], together with new theoretical simulations based on mode matching and circuit theory, are shown in Fig. 3 (for a description of the experimental setup, see Fig. 1(d) and [30, Fig. 1]).

Note that a transmission maximum is observed at certain frequency $(11.42 \mathrm{GHz})$ that is well below the resonance frequency expected for the isolated rectangular aperture (about $15 \mathrm{GHz}$ ). This experimental frequency of the transmission maximum is close and below the cutoff frequency of the $\mathrm{TM}_{01}$ mode $\left(f_{1}=\right.$ $11.768 \mathrm{GHz}$ for the nominal dimensions of the employed circular waveguide, $r=9.75 \mathrm{~mm}$ ). As predicted by our model, the transmission peak is immediately followed by a transmission dip at the cutoff frequency of $\mathrm{TM}_{01}$ mode ( $C$ becomes singular at that frequency). This transmission dip is the so-called Rayleigh-Wood's anomaly in the frame of the theory of periodic gratings. The above behavior is the same reported in many previous papers for 2-D arrays of holes (experiments and simulations). The reason for this analogy is that the underlying models provided by our theory are very close for those, in principle, physically different problems.

It was previously mentioned that the circular waveguide with an off-centered diaphragm is a convenient geometry to illustrate the significance of our theory. This point might deserve some additional clarification, and in particular, it should be clarified why the rectangular (or square) waveguides proposed in [31] or [28] have not been used. The first reason is that those cross sections are not appropriate to clearly show our point that impedance matching is an apparent physical fact different from periodicity: the boundary conditions at rectangular (or square) waveguide walls could be (theoretically) reproduced using suitable multiple plane-wave excitation of a periodic 2-D distribution of holes. Thus, it could be reasonably argued that no new physics would appear in our approach. There is, however, another important practical issue: the first higher order mode that can be involved in extraordinary transmission in the rectangular 

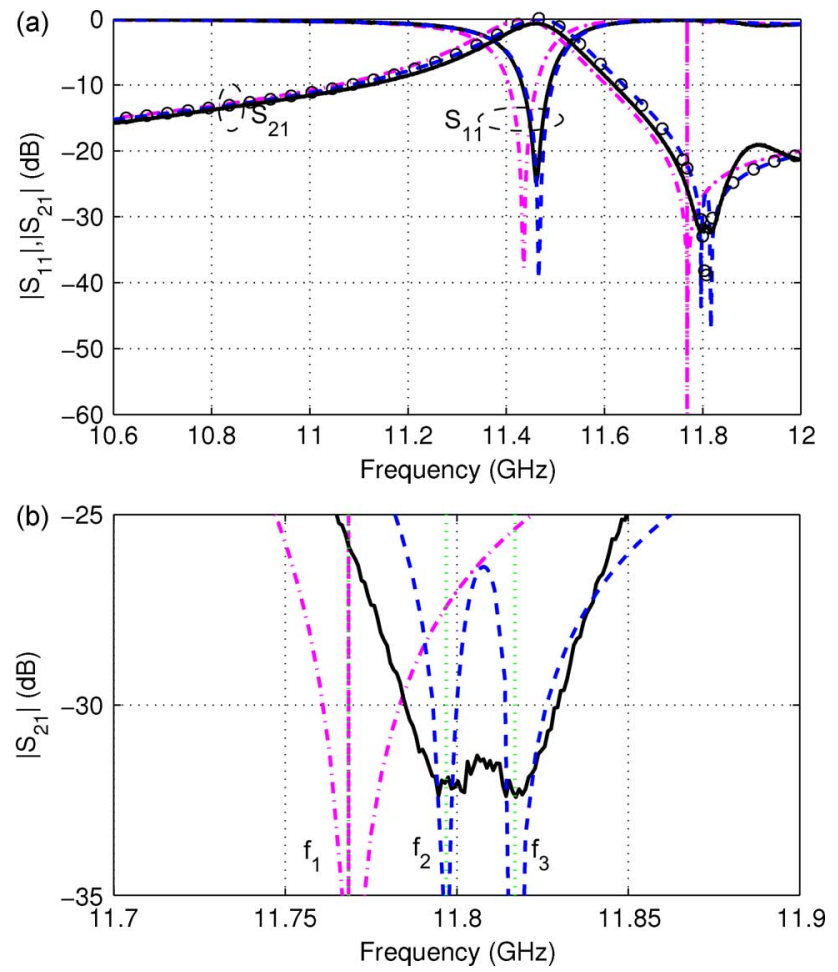

Fig. 3. Extraordinary transmission with a rectangular hole: experiment (solid line; black in online version) and theory (full-wave mode-matching results) with nominal waveguide dimensions (dash dotted line; pink in online version) and when slightly different circular cross-section waveguides, compatible with fabrication tolerances, are considered (dashed line; blue in online version). Circles correspond to the circuit model predictions with parameters extracted from a few full-wave points. (a) Transmission $\left(\left|S_{21}\right|\right)$ and reflection $\left(\left|S_{11}\right|\right)$ coefficients. (b) Detail of the transmission dip frequency region. Dimensions (in millimeters) as in Fig. 1: $r=9.75$ (nominal), $a=10, b=2, s=7, t=0.35$. $r_{1}=9.71, r_{2}=9.73$ (assumed values of waveguide radii to account for the two closely spaced transmission zeros).

(or square) section case is the $\mathrm{TM}_{11}$ mode (the $\mathrm{TM}_{12}$ mode is used in [28] because the authors considered a centered hole). Since there would always be two propagating modes below the cutoff frequency of the $\mathrm{TM}_{11}$ mode, it had precluded the desired single-mode operation regime because any small discontinuity (including excitation probes) would have excited the two propagating modes. In such a case, the interpretation of the results, and of the analogy with the original 2-D periodic problem, would be rather difficult. A third positive feature of the use of circular waveguide is that standard rectangular to circular section transitions are available. Before performing experiments, the whole system (coax-to-rectangular waveguide transitions, rectangular to circular mode converters, and the circular waveguide sections) was tested for the possibility of spurious reflections. Very satisfactory return losses below $30 \mathrm{~dB}$ were measured in the absence of the diaphragm, which confirms the suitability of the present setup for our purposes.

\section{B. Transmission Dips}

As already mentioned, the main results described above were published in [30], where good agreement between experiments and theory (mode matching and circuit modeling) was reported. In the following, we will extend our previous research on this topic and will present new phenomenology and discussions.
One of the new features come from the close observation of the experimental transmission dips reported in [30, Fig. 2]. In particular, it can be observed that instead of a single transmission dip, there are two closely spaced transmission dips. The details of this part of the experimental transmission spectrum is now reproduced in Fig. 3(b). In order to explain this unexpected observation with our theory, we can argue that, in the case of periodic structures, the waveguides assumed in our model at both sides of the discontinuity are virtual and, therefore, they are identical. However, the circular waveguides of our experimental setup are unlikely identical (due to fabrication tolerances). Thus, the cutoff frequencies of the $\mathrm{TM}_{01}$ modes at both sides of the diaphragm might be slightly different. According to our theory, it implies that the capacitance at both sides of the discontinuity will be singular at two slightly different frequencies, which would coincide with the cutoff frequencies of the $\mathrm{TM}_{01}$ modes of the different circular waveguides: $f_{2}=11.797 \mathrm{GHz}$ and $f_{3}=11.817 \mathrm{GHz}$, respectively (labels used in Fig. 3(b) and other subsequent figures). Between the two singular points, the overall capacitance has large, but finite values. Therefore, our model predicts that the waveguide radii of the two waveguide sections at both sides of the diaphragm should actually be 9.73 and $9.71 \mathrm{~mm}$, which is utterly within the fabrication tolerances. When these radii are used in mode-matching simulations (blue lines in online version of Fig. 3), the whole transmission spectrum is captured with excellent agreement. Fig. 3(a) also shows that the slight discrepancy between experimental and theoretical transmission peaks, corresponding to the nominal value of $r$, completely disappears in the new simulations. Since these waveguides have been used in subsequent experiments, these radii will be used in Section II-C.

\section{Elliptical Aperture}

In addition to the rectangular shape, an elliptical aperture slightly larger than the rectangular one was built and measured. The comparison between experimental results, mode matching results, and circuit theory data are given in Fig. 4. Very good agreement is found again between theory and experiment provided the same circular waveguide radii used in Fig. 3 are used here. It can then be concluded that the circuit model accurately accounts for the behavior of the aperture regardless of its shape, provided this shape is relatively simple (complex patterns such as Jerusalem crosses, fractal geometries, etc., are clearly expected to require more sophisticated circuit models). When comparing these results with those corresponding to the rectangular hole, it can be observed that the transmission dips are not affected by the change of shape and size of the hole. This is consistent with the fact that the cutoff frequency of the $\mathrm{TM}_{01}$ mode is actually what controls these dips. However, the transmission peak is shifted to lower frequencies and the bandwidth is now larger. This transmission peak can still be called extraordinary because the expected transmission peak, in the absence of the circular waveguide environment, should be close to the onset frequency of the first mode of the elliptical waveguide forming the hole (in this case, $13.827 \mathrm{GHz}$ ). It is then the influence of the $\mathrm{TM}_{01}$ mode of the circular waveguide what yields the frequency downshift. From the circuit point of view, this result can easily be explained by the expected higher value 

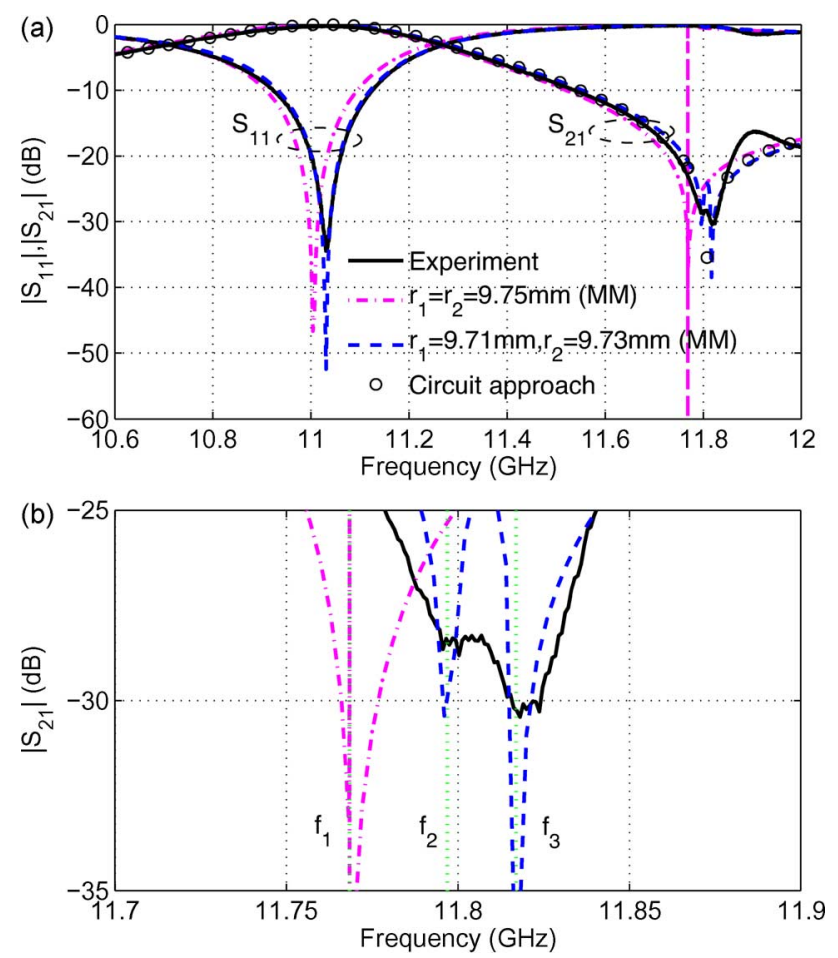

Fig. 4. Extraordinary transmission with an elliptic hole: experiment and theory (mode-matching results: MM). (a) Transmission $\left(\left|S_{21}\right|\right)$ and reflection $\left(\left|S_{11}\right|\right)$ coefficients. (b) Detail of the zero transmission frequency region. Dimensions (in millimeters) as in Fig. 1: $r=9.75$ (nominal), $a_{x}=13, a_{y}=3, s=6$, $t=0.35, r_{1}=9.71$, and $r_{2}=9.73$ (actual values).

of the inductance provided by the elliptical hole, in comparison with that of the rectangular hole, due to the slightly larger size of the elliptical hole. The extracted parameters for the circuit model confirm this qualitative prediction: $L=0.34 \mathrm{nH}$ for the rectangular aperture and $L=0.61 \mathrm{nH}$ for the elliptical one. In this situation, the capacitance required to reach the resonance condition of the $L C$ tank in Fig. 2(a) is smaller for the elliptical hole. The resonance can then be obtained at a frequency not too close to the singularity (cutoff frequency of the $\mathrm{TM}_{01}$ mode). The system can be viewed as an $L C$ resonator loaded by the transmission lines shown in Fig. 2(a). The resonance frequencies of the rectangular and elliptical holes are not very different, but the capacitance of the rectangular hole is meaningfully larger than that the elliptical aperture (the reverse happens for the inductance). In such a case, conventional circuit theory predicts a smaller 3-dB bandwidth for the resonator associated with the rectangular aperture, as it is clearly observed in the simulations and measurements of Figs. 3(a) and 4(a). Thus, all the main features of the observed transmission spectrum can be anticipated using the circuit model in a rather trivial manner.

\section{INFLUENCE OF LOSSES ON EXTRAORDINARY TRANSMISSION}

In this section, we will show the effect of conductor losses on the transmission and reflection coefficients, and how these effects can be accounted for by our equivalent-circuit model. From the experimental results reported in [30], and also those in Fig. 4, it can be concluded that losses give rise to a slight reduction in the transmitted power without any meaningful effect on the reflection coefficient. Moreover, losses are smaller for the elliptical hole (0.25-dB insertion loss against $0.75 \mathrm{~dB}$ for the rectangular aperture), which has a resonance frequency lower than that of the rectangular hole. Among other possible alternatives, conductor losses can be included in the circuit model by simply adding a resistor connected in series to the inductance (we ignore propagation losses along the nonideal circular waveguide sections because of their negligible contribution). After doing this, the effective impedance $Z_{\text {res }}$ of the $L C$-tank resonator in Fig. 2(a) at the theoretical resonance frequency $\left(\omega_{0}=1 / \sqrt{L C}\right)$ is given by

$$
Z_{\text {res }}=\sqrt{\frac{L}{C}}\left[\frac{\sqrt{L / C}}{R}-\mathrm{j}\right]
$$

where $R$ represents the skin-effect resistance of the metal plate with the hole. Some incremental inductance should have also been added, but it is assumed to be negligible in comparison with $L$.

The above expression tells us that the impedance of the resonator at resonance is infinite when $R=0 \Omega$ (perfect conductor case). This is true for any pair of values of $L$ and $C$, which means that total transmission peaks are predicted for the lossless case below the cutoff frequency of the $\mathrm{TM}_{01}$ mode for any size of the hole (i.e., for any value of its inductance). Small holes would provide very low inductance values, thus requiring large values of $C$ to yield resonance. These high values of $C$ can only be reached at frequencies very close to Rayleigh-Wood's anomaly. In such circumstances, the transmission peak would also be extremely narrow, although it could be observed by a sufficiently fine frequency scan. This fact has been verified in many full-wave simulations reported in the literature (see, for instance, [24] for a circular aperture inside a cylindrical waveguide). However, the presence of losses can dramatically modify the above scenario.

If losses are actually low, we can expect some slight attenuation, such as that shown in the experimental results in Figs. 3(a) or 4(a). The meaning of low losses in this context strongly depends on the size of the hole for a given frequency range and metal choice. For instance, the cases treated in Fig. 4 or in [30] can be considered as "low losses" cases. The holes, although sub-wavelength in size, are large enough to have relatively high values of inductance. In such a case, the ratio $\sqrt{L / C} / R$ in (1) is large enough to make $\left|Z_{\text {res }}\right| \gg Z_{\mathrm{TE}_{11}}$. Impedance matching is, therefore, still quite good at resonance, and low reflection is obtained. Absorption is weak because the impedance of the circuit branch with $R$ and $L$ is relatively large, thus avoiding high currents at resonance. It should be noticed that current in the circuit model accounts for the level of surface current flowing over the metal plate in the physical system. Roughly speaking, holes with relatively large dimensions (along the direction perpendicular to the impinging electric field) have large inductances. In such a case, current flowing through the resistance (i.e., the surface current over the metal plate) is relatively small if compared with the current flowing in the case of smaller holes. Large holes are, therefore, related to good matching and low losses. If the dimension of the hole along the direction perpendicular to the polarization of the incident field is reduced, current in the $L C$-tank cir- 


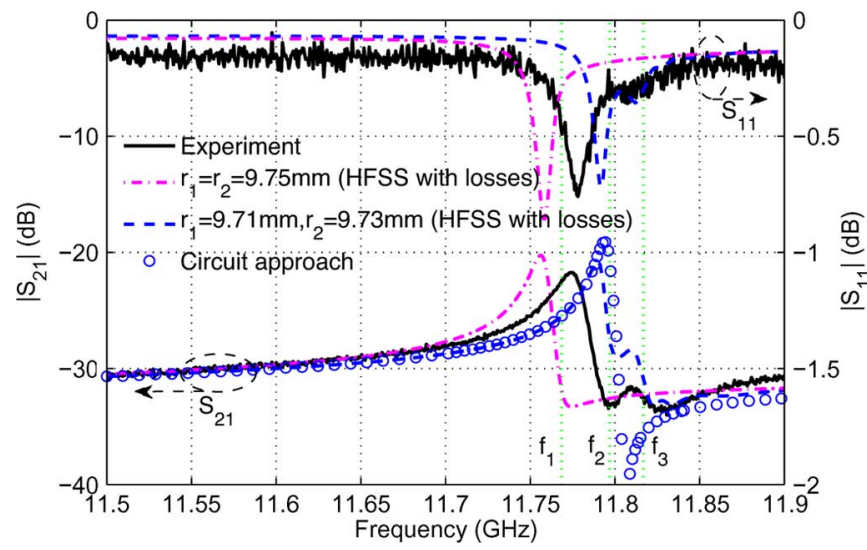

Fig. 5. Experimental, numerical, and analytical results (including losses) for the transmission $\left(\left|S_{21}\right|\right)$ and reflection $\left(\left|S_{11}\right|\right)$ coefficients of a rectangular hole of very small size. Dimensions (in millimeters): $r=9.75, a=5.7, b=2$, $s=7$, and $t=0.35$. Numerical results with losses are obtained with Ansoft Corporation's HFSS considering a surface boundary condition with metal conductivity of $\sigma=10^{6} \mathrm{~S} / \mathrm{m}$.

cuit (and, therefore, ohmic losses) automatically increases due to the smaller value of the impedance of the inductive and capacitive branches of the resonator (if reasonable matching is assumed, the driving voltage is similar for large and small holes). Certainly, higher current through $R$ yields higher losses. Note that the current in the resonator can be much larger than that in the transmission lines at both sides of the discontinuity (this latter current is associated with the value of the magnetic field of the incident and transmitted waves) if the holes are small enough (low inductance holes). This predicted current crowding for small holes is fully consistent with the reported field enhancement over the perforated plate at extraordinary transmission frequencies in periodic systems. In many papers, this field enhancement is actually considered the main reason for extraordinary transmission [1], [3]; total transmission, though, is still possible without field enhancement if the apertures are large enough (indeed, this is the case for conventional frequency selective surfaces). Low inductance values, however, are responsible for another important effect. If resonance is achieved with low inductance and high capacitance, $\left|Z_{\text {res }}\right|$ in (1) can be of the same order of magnitude or even smaller than $Z_{\mathrm{TE}_{11}}$, thus giving place to strong mismatching. Our model not only predicts strong material losses, but also strong mismatching for small holes in lossy walls.

For small enough holes, the previous rationale suggests that poor transmission, due to strong mismatching and absorption, should be expected when the theoretical (lossless) extraordinary transmission frequency is very close to Rayleigh-Wood's anomaly. This prediction has been experimentally verified by using a rectangular diaphragm having almost half of the length of the aperture used in [30] or, in this paper, in Fig. 3. No new iris was actually built; instead, we partially obturated the original $10-\mathrm{mm}$ aperture with adhesive copper sheets and measured the resulting slit dimensions using a microscope. It results on an effective conductivity meaningfully smaller than that of the aluminium employed to fabricate the irises. The value of this effective conductivity was estimated from the comparison of HFSS simulations with measured data. The results are shown in Fig. 5, which include simulated (HFSS), measured, and circuit-theory

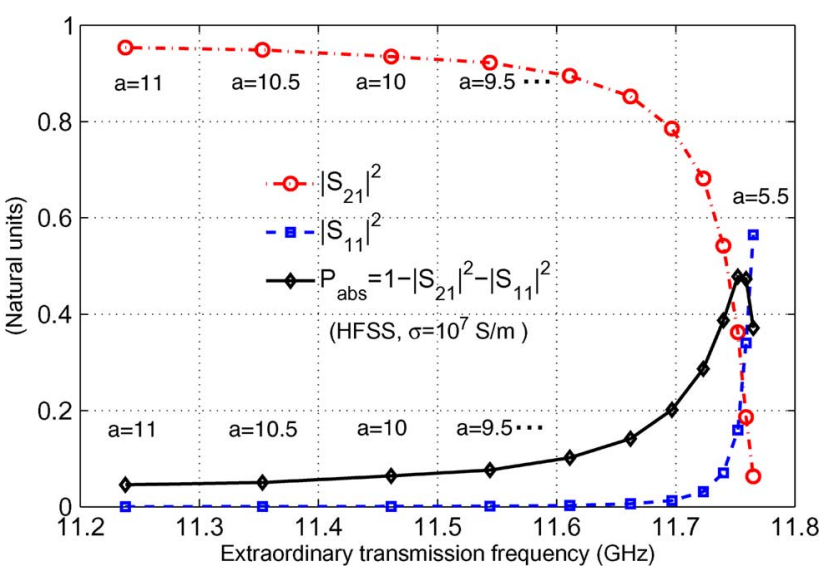

Fig. 6. Calculated (HFSS) values of transmitted $\left(\left|S_{12}\right|^{2}\right)$, reflected $\left(\left|S_{11}\right|^{2}\right)$, and absorbed $\left(1-\left|S_{11}\right|^{2}-\left|S_{12}\right|^{2}\right)$ power for 12 different rectangular holes made in a thin conductive plate of conductivity $\sigma=10^{7} \mathrm{~S} / \mathrm{m}$. Dimensions of the holes: $b=2 \mathrm{~mm}, a=5.5+(n-1) 0.5 \mathrm{~mm}(n=1,2, \ldots, 12)$. The waveguide dimensions are the same as in previous figures.

data. Simulated data have been obtained using the nominal radii of the waveguides and the radii deduced from the transmission zeros. The conductivity used in HFSS simulations was $10^{6} \mathrm{~S} / \mathrm{m}$ for the plate with the iris. The ohmic resistance in the circuit model was computed by just allowing the inductance to be complex (its imaginary part is trivially related to the resistance). It is remarkable how the circuit model perfectly captures the details of the spectrum for the lossy case when very small holes are involved. Note that a weak transmission peak, very close to Rayleigh-Wood's anomaly, can be clearly seen for the transmission coefficient $S_{12}$. Impedance matching is very poor, as it is clear from the reflection coefficient $S_{11}$. Moreover, an important fraction of the power is dissipated by the perforated metal plate (dissipated power is readily computed as $1-\left|S_{11}\right|^{2}-\left|S_{12}\right|^{2}$ ). In this particular example, $84.1 \%$ of the incident power is reflected, $0.6 \%$ is transmitted, and $15.3 \%$ is dissipated. From the circuit theory point of view, the observed spectrum can easily be explained. For the extracted values of $L, C$, and $R, Z_{\text {res }}$ in (1) is quite small in comparison with $Z_{\mathrm{TE}_{11}}$, which causes very poor matching even at resonance. The practical important consequence of this fact is that we cannot have high transmission peaks at frequencies too close to Rayleigh-Wood's anomaly due to the limitation imposed by ohmic losses (i.e., we cannot have "extraordinary transmission" for deeply sub-wavelength holes in lossy walls). This is an important drawback for the practical applications of the potential highly selective properties that extraordinary transmission could provide at microwave or millimeter-wave frequencies. In order to have a quantitative idea about the severity of this limitation, we have studied the transmission properties of our waveguide system for various sizes of the rectangular aperture. The results have been obtained using the HFSS simulator using a conductivity of $\sigma=10^{7} \mathrm{~S} / \mathrm{m}$. Transmitted, reflected, and absorbed power (at the maximum transmission frequency point) are shown in Fig. 6 for a number of rectangular holes having different widths ( $a$ ranging from 11 to $5.5 \mathrm{~mm}$ in steps of $0.5 \mathrm{~mm}$ ). Transmissivity (red line in online version), which should reach unity in a lossless system, strongly degrades for values of $a$ below $8.0 \mathrm{~mm}$ due to both absorption and mismatching. This clearly indicates that the degree of 
squeezing of electromagnetic waves into small apertures is severely limited by the presence of actual conductor losses.

At this point, it should be highlighted that the above discussion on the effects of losses in the circular waveguide problem also applies to 2-D periodic distributions of holes. Thus, it would be very difficult to observe a genuine extraordinary transmission peak in common experiments with frequency-selective surfaces at microwave frequencies unless the experiment is suitably designed for that purpose, as was done in [6] (the experimental results reported in that paper give us a good opportunity to clarify our analysis). In [6], the authors studied several perforated aluminium screens having various thicknesses. Though our analysis up to now is only valid for very thin screens (thick screens is the subject of Section IV), the qualitative predictions concerning the role of losses is quite similar for thin and thick screens, provided that the thickness is not too large. In [6], the lattice constant (namely, the size of the square unit-cell waveguide as defined, for instance, in [26]) was $d=5 \mathrm{~mm}$ and the cylindrical holes have radii $r=1.25 \mathrm{~mm}$ and $1.0 \mathrm{~mm}$. For $r=1.25 \mathrm{~mm}$, the situation is very close to the experimental situation studied in this paper, and more specifically, to the elliptical diaphragm case. The parameters relevant to establishing the comparison is the ratio of the cutoff frequency of the TM mode involved in the extraordinary transmission $\left(\mathrm{TM}_{01}\right.$ mode in the case of the circular waveguide and $\mathrm{TM}_{02}$ mode of the square cross-section parallel-plate waveguide in [6]) and the cutoff of the fundamental mode of a hollow pipe waveguide having the same cross section as the hole (elliptical hole in our case and circular hole in [6]). For $r=1.25 \mathrm{~mm}$, this ratio is 0.85 in [6] and 0.853 in our experiment. Looking at the experimental results in [6, Fig. 3] for the thinnest screen $(t=0.4 \mathrm{~mm})$ and $r=1.25 \mathrm{~mm}$, almost total transmission is observed for a wavelength that is about 1.04 times the cutoff wavelength of the $\mathrm{TM}_{02}$ mode of the unit cell waveguide. Our results for the elliptical hole give almost total transmission at a wavelength that is 1.07 times that of the $\mathrm{TM}_{01}$ mode of the circular waveguide. The case $r=1.0 \mathrm{~mm}$ in [6], however, corresponds to an extraordinary transmission frequency much closer to Rayleigh-Wood's anomaly (in this case, from the inset in [6, Fig. 2], the expected extraordinary transmission wavelength to cutoff wavelength ratio is 1.012). In the frame of our model, this ratio decrease comes from the smaller inductance provided by the hole, which should yield poor impedance matching and higher losses. This is clearly what is found in the experimental results reported in [6, Fig. 3]: transmission for $r=1.25 \mathrm{~mm}$ was $95 \%$, while less than $17 \%$ is measured for a slightly smaller hole $(r=1.0 \mathrm{~mm})$. These experimental results are fully consistent with our predictions and results in spite of dealing with different systems. Again, the reason is that the underlying physics is basically the same.

\section{EFFECTS OF THE DiAPHRAGM THICKNESS}

In many simulations of extraordinary transmission through 2-D periodic arrays of holes in metallic walls with nonnegligible thickness, the existence of two transmission peaks, rather than a single one, has been reported. A detailed study of the double peak and its dependence on screen thickness can be found in [5, Fig. 4] or [3, Fig. 8], although this double peak also appears in many other papers [6], [39]. Nevertheless, to the best of the authors' knowledge, no experimental evidence of this double peak phenomenon has been reported in the literature, which is probably due to its high sensitivity to actual losses. Our equivalent-circuit approach certainly predicts the existence of such a double peak in the lossless case; it also provides a simple explanation of why losses destroy one of those peaks. In [26], some modifications of the simple $L C$-tank circuit model was proposed with the purpose of incorporating the effect of the screen thickness (2-D arrays of holes case). This model successfully predicts the double peak, as well as the dependence of the position of the peaks with respect to the screen thickness. Actually, two different circuits were proposed in [26]. The simpler one used a $\pi$-circuit with three inductances, a series inductance $L_{s}$, and two shunt inductances $2 L_{p}$, which account for the reactive energy stored inside the hole by the dominant TE modes below cutoff. This circuit model is adapted here to deal with the expected double peak phenomenon in the circular waveguide with a thick diaphragm. The circuit is represented in Fig. 2(b). The $\pi$-circuit made of inductors is the simplest way of representing TE modes below cutoff inside a waveguide section. The only difference with the case treated in [26] is that the propagating modes in the input and output waveguides are different from those considered in [26]. In spite of those minor differences, it is clearly expected that the qualitative behavior of the transmission spectrum of both problems should be the same, provided that the circular waveguide sections operate in the single-mode regime. The parameters of this model can once again be extracted from numerical/experimental results for just three arbitrarily chosen frequency points (see Appendix B).

The behavior of transmission peaks with respect to the diaphragm thickness is shown in Fig. 7(a) for some particular cases ignoring losses. As expected, the type of transmission spectrum is qualitatively identical to that obtained for periodically perforated structures. It is worth mentioning how the nonzero thickness of the perforated plate makes the extraordinary transmission frequencies shift toward the Rayleigh-Wood's anomaly. For instance, the total transmission frequency for the zero thickness screen in Fig. 3 is around $11.42 \mathrm{GHz}$ for a rectangular hole with dimensions $a=10.0 \mathrm{~mm}$ and $b=2.0 \mathrm{~mm}$; the same aperture in a thick $5.0-\mathrm{mm}$ screen has a peak around $11.7 \mathrm{GHz}$. This is easily understandable from the circuit point of view: the internal inductance of the hole open cavity is shunt connected with the external inductance of the TE modes in the circular waveguide. The resulting global inductance is meaningfully smaller than that of the zero-thickness screen case. This inductance decrease explains the shift to higher frequencies of the transmission peak. Fig. 7(a) also shows the evolution of the two transmission peaks as the thickness increases. The peaks get closer and closer as the thickness is larger and larger, in perfect agreement with the results reported in [3], [5], and [26]. For very thick screens, no peak is observed because of the exponentially vanishing interaction between the two sides of the diaphragm.

The influence of losses can be appreciated in Fig. 7(b), which shows HFSS simulations including ohmic metal losses (in this case, a typical effective conductivity of $10^{7} \mathrm{~S} / \mathrm{m}$ for a not polished aluminium surface has been used). These simulations show how dissipation and mismatching cause certain 

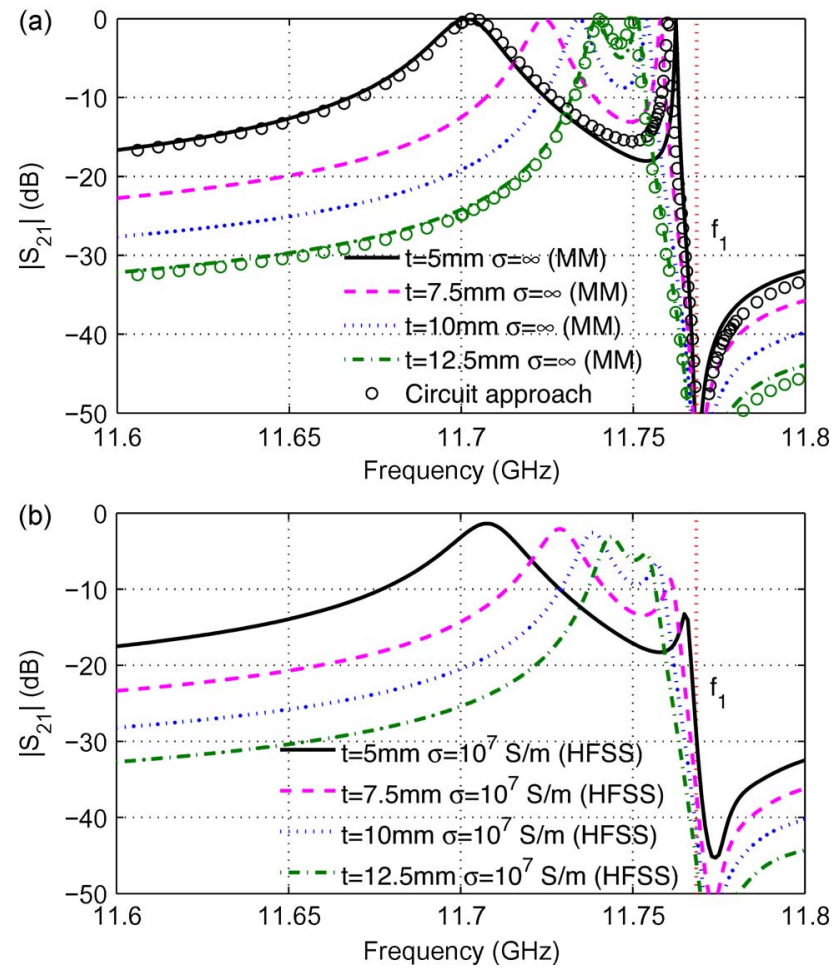

Fig. 7. Transmission coefficient $\left(\left|S_{21}\right|\right)$ of the rectangular hole for different values of the metal thickness $t$ (other dimensions in millimeters are: $r=9.75$, $a=5.7, b=2$, and $s=7$ ). (a) Lossless case (accurate mode-matching results). (b) Same geometry considering metal losses (HFSS data). Circles denote circuit model results.

attenuation in the wider bandwidth peak, while the second peak (always very narrowband and close to Rayleigh-Wood's anomaly) almost completely disappears, in agreement with our qualitative predictions. Obviously, a similar behavior should be expected for extraordinary transmission systems based on periodic structures. Thus, typical levels of metallic losses would preclude a clear observation of the second peak. Superconducting screens, supported by low-loss dielectrics, could be used for the observation of the second peak.

\section{Higher ORDER FieldS AROUND EXTRAORDINARY-TRANSMISSION FREQUENCY}

In this section, the modal field distributions in the neighborhood of the extraordinary transmission and Rayleigh-Wood's anomaly frequencies will be studied. For the case of a rectangular hole, Fig. 8 shows the transmission coefficients of the transmitted propagating $\mathrm{TE}_{11}$, and evanescent $\mathrm{TM}_{01}$ modes, referred to the incident $\mathrm{TE}_{11}$ mode. The amplitude coefficients are computed at an output reference plane placed at a distance $d=10 \mathrm{~mm}$ from the hole plane, as shown in Fig. 9. It can be seen in Fig. 8(a) that the transmission coefficient of both modes reaches a maximum at the corresponding total transmission frequencies. The amplitude of the fundamental mode and the first higher order mode are quite similar for the total transmission frequencies that are very close to the Rayleigh-Wood's anomaly frequency. The relative amplitude of the $\mathrm{TM}_{01}$ mode, however, exponentially increases as the total transmission frequency approaches to Rayleigh-Wood's anomaly. For instance,
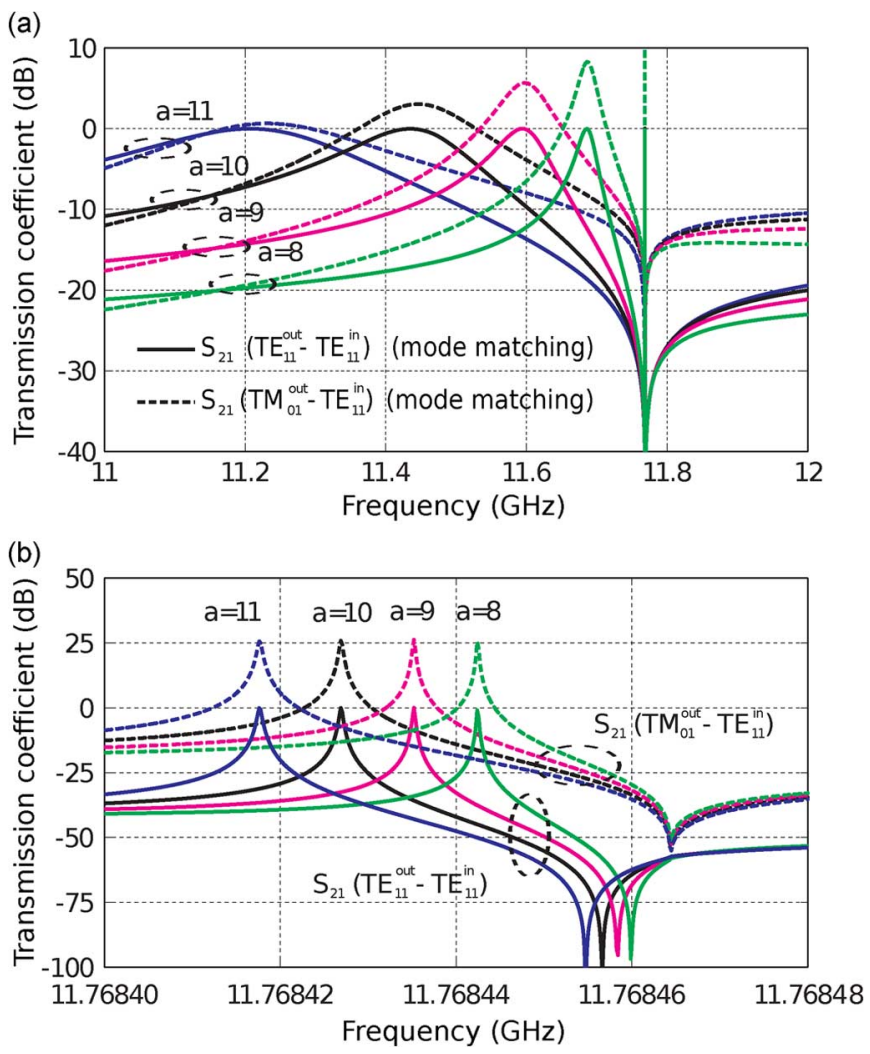

Fig. 8. Transmission coefficient (obtained by mode matching) of the rectangular hole for different values of the rectangular aperture $a$ (other dimensions in millimeters are: $r=9.75, b=2, s=7$, and $t=0.35$ ). (a) Transmission coefficients, referred to the incident $\mathrm{TE}_{11}$ mode, of the $\mathrm{TE}_{11}$ and $\mathrm{TM}_{01}$ modes at the output waveguide (reference plane at a distance $d=10 \mathrm{~mm}$ of the hole plane). (b) Detail of the frequency region close to the Rayleigh-Wood's anomaly.

for $a=10.0 \mathrm{~mm}$, the amplitude level of the fundamental mode and first higher order mode are quite similar $(10 \mathrm{~mm}$ away from the hole plane); for $a=8.0 \mathrm{~mm}$, the amplitude ratio is more than $8 \mathrm{~dB}$ in favor of the evanescent mode. It is also worth studying the amplitude of higher order modes (higher than $\mathrm{TM}_{01}$ ) in this structure. Table I shows the amplitudes of the first few higher order modes supported by the circular waveguides for four different rectangular apertures. These amplitudes are evaluated at the extraordinary transmission frequency. It is clear that higher order mode amplitudes are very small and almost independent of the size of the aperture, except for the $\mathrm{TM}_{01}$ mode, which apparently dominates the field distribution when the aperture is electrically small.

A detailed inspection of the frequency region corresponding to the second peak (lossless case), which is extremely close to Rayleigh-Wood's anomaly, is shown in Fig. 8(b). This plot shows more than $25 \mathrm{~dB}$ of difference between the two transmission coefficients at the total transmission frequencies. (This difference would obviously be larger at the discontinuity plane due to the evanescent nature of $\mathrm{TM}_{01}$ within the considered frequency range.) This fact is a clear example of a huge field enhancement associated with extraordinary transmission in our closed waveguide, similar to that reported in the literature for the extraordinary optical transmission through periodic holey screens [3]. 


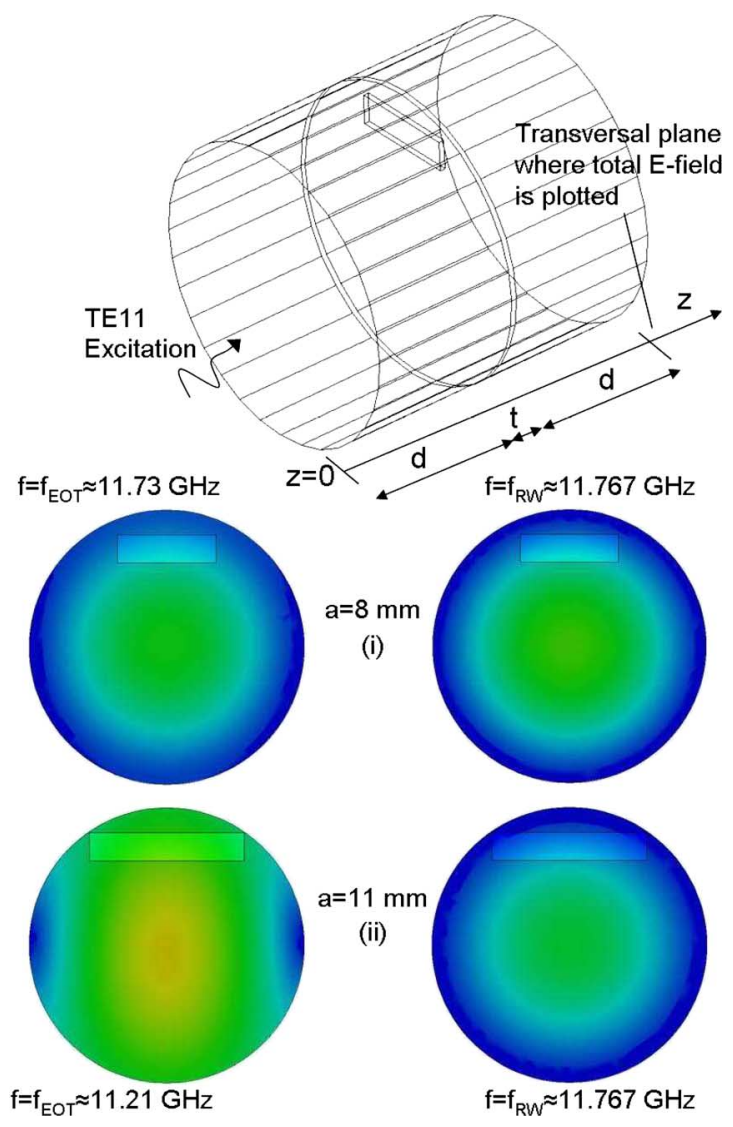

Fig. 9. Electric field pattern for frequencies close to the total transmission $(f \approx$ $\left.f_{\mathrm{EOT}}\right)$ and to the Rayleigh-Wood's anomaly $\left(f \approx f_{\mathrm{RW}}\right)$ for two rectangular apertures of size: (i) $a=8 \mathrm{~mm}$ and (ii) $a=11 \mathrm{~mm}$.

TABLE I

RELATIVE AMPLITUDES (IN DECIBELS) OF INCIDENT AND Higher ORDER MODES FOR SEVERAL VALUES OF APERTURE WIDTH. $b=2.0 \mathrm{~mm}$

\begin{tabular}{c|c|c|c|c|c|c}
\hline$a(\mathrm{~mm})$ & freq $(\mathrm{GHz})$ & $\mathrm{TE}_{11}$ & $\mathrm{TM}_{01}$ & $\mathrm{TE}_{21}$ & $\mathrm{TM}_{11}$ & $\mathrm{TE}_{21}$ \\
\hline \hline 11.0 & 11.210 & 0.00 & 0.57 & -15.62 & -26.89 & -28.24 \\
10.0 & 11.435 & 0.00 & 2.98 & -15.36 & -26.73 & -27.78 \\
9.0 & 11.594 & 0.00 & 5.64 & -15.13 & -26.67 & -27.35 \\
8.0 & 11.686 & 0.00 & 8.28 & -14.96 & -26.76 & -26.96 \\
\hline
\end{tabular}

Electric field distributions, obtained by HFSS simulations, are plotted in Fig. 9 for the total transmission and Rayleigh-Wood's anomaly frequencies [maximum peaks and transmission dips in Fig. 8(b)]. If the aperture is small, Fig. 9(i) shows that the fields are dominated by the $\mathrm{TM}_{01}$ mode (with no circumferential variation in the field) at a distance of $10.0 \mathrm{~mm}$ from the discontinuity plane. This situation changes for larger holes, at least at the total transmission frequency. On the left-hand side of Fig. 9(ii), it is apparent that the field distribution is dominated by the $\mathrm{TE}_{11}$ mode (with vertical polarization). Nevertheless, around the Rayleigh-Wood's anomaly frequency, fields are, again, totally dominated by the $\mathrm{TM}_{01}$ mode. This is because there is no meaningful attenuation of the high density fields associated with such mode so close to cutoff. It is interesting to note that the magnitude of the total electric field has a peak at total transmission frequencies (total fields at Rayleigh-Wood's anomaly point are small) and increases as the total transmission frequency approaches the
Rayleigh-Wood's anomaly. It could be an interesting way of obtaining high levels of field concentration, as required in some optical applications. Unfortunately, we have already shown that real losses will drastically reduce the field level. Nevertheless, significant field enhancement is still expected even in lossy systems, being more important at optical frequencies, where metals are not ohmic conductors, but lossy plasmas.

\section{CONCLUSIONS}

This paper has presented a detailed experimental and theoretical study of the transmission properties of small diaphragms placed inside a hollow pipe circular waveguide. The analogy existing with extraordinary transmission observed in periodically perforated screens has been highlighted. If emphasis is put on impedance matching rather than on surface wave excitation, a unified theory can be used to explain both physical situations. The resonant behavior of near fields around the aperture are responsible for impedance matching and, thus, extraordinary transmission in both problems. The theoretical and experimental study of the role of material losses reported in this paper leads to the conclusion that very small holes in real metal screens would yield poor transmission due to strong mismatching and absorption. The circuit model arising from our analysis has simplified the physical interpretation of the experimental results. This model also helps to extract conclusions about what actually can be expected from extraordinary transmission systems and about how to improve their electrical behavior for specific purposes with potential practical applications.

\section{APPENDIX A}

The expression given in [26, eq. (4)] can readily be adapted to the circuit in Fig. 2(a) to give

$$
\frac{\mathrm{j} 2 \omega}{S_{12}}=-\left(\frac{C}{Y_{\mathrm{TE}_{11}}}\right) \omega^{2}+\mathrm{j} 2 \omega+\frac{1}{L Y_{\mathrm{TE}_{11}}} .
$$

Assuming the following frequency dependence of the capacitance [26]:

$$
C(\omega)=C_{0}+\frac{A}{\sqrt{\omega_{c}^{2}-\omega^{2}}}
$$

where $\omega_{c}$ is the cutoff frequency of the first higher order mode $\left(\mathrm{TM}_{01}\right.$ in the case under study), (2) can be rewritten as

$$
\omega C_{0}+\frac{\omega}{\sqrt{\omega_{c}^{2}-\omega^{2}}} A-\frac{1}{\omega} L^{-1}=\mathrm{j} 2 Y_{\mathrm{TE}_{11}}\left[1-\frac{1}{S_{12}}\right] .
$$

If we write the above equation for three different frequency values, the following linear system of equations will be obtained:

$$
\begin{aligned}
\omega_{i} C_{0}+ & \frac{\omega_{i}}{\sqrt{\omega_{c}^{2}-\omega_{i}^{2}}} A-\frac{1}{\omega_{i}} L^{-1} \\
& =\mathrm{j} 2 Y_{\mathrm{TE}_{11}}\left(\omega_{i}\right)\left[1-\frac{1}{S_{12}\left(\omega_{i}\right)}\right], \quad i=1,2,3
\end{aligned}
$$

whose solution gives us the values of unknown parameters $C_{0}$, $A$, and $L$ 


\section{APPENDIX B}

If the even/odd $(e / o)$ excitation method is applied to the transmission line circuit shown in Fig. 2(b), it is found that the reflection coefficient corresponding to the even/odd excitation [see Fig. 2(c)] is given by

$$
S_{11}^{e / o}=\frac{\mathrm{j} 2 \omega L_{e / o}^{\mathrm{eq}} Y_{\mathrm{TE}_{11}}-\left(1-\omega^{2} L_{e / o}^{\mathrm{eq}} C\right)}{\mathrm{j} 2 \omega L_{e / o}^{\mathrm{eq}} Y_{\mathrm{TE}_{11}}+\left(1-\omega^{2} L_{e / o}^{\mathrm{eq}} C\right)}
$$

where

$$
L_{e / o}^{\mathrm{eq}}=2 L \| L_{e / o}
$$

Considering now the even excitation, we see that $S_{11}^{e}$ can readily be obtained from the numerically computed reflection and transmission coefficient of the whole system as

$$
S_{11}^{e}=S_{11}+S_{12}
$$

Expression (6) can be rewritten as

$$
\frac{1}{\omega}\left(L_{e}^{\mathrm{eq}}\right)^{-1}-\omega C=\mathrm{j} 2 Y_{\mathrm{TE}_{11}} \frac{1-S_{11}^{e}}{1+S_{11}^{e}}
$$

which taken into account the explicit frequency dependence of $C$ given in (3) can be cast in the following way:

$$
\frac{1}{\omega}\left(L_{e}^{\mathrm{eq}}\right)^{-1}-\omega C_{0}-\frac{\omega}{\sqrt{\omega_{c}^{2}-\omega^{2}}} A=\mathrm{j} 2 Y_{\mathrm{TE}_{11}} \frac{1-S_{11}^{e}}{1+S_{11}^{e}} .
$$

This last expression makes it possible to write the following linear system of equations to find the values of the unknown parameters $C_{0}, A$, and $L_{e}^{\mathrm{eq}}$ from just three numerical evaluations of $S_{11}^{e}$ at different frequencies:

$$
\begin{aligned}
& \frac{1}{\omega_{i}}\left(L_{e}^{\mathrm{eq}}\right)^{-1}-\omega_{i} C_{0}-\frac{\omega_{i}}{\sqrt{\omega_{c}^{2}-\omega_{i}^{2}}} A \\
&=\mathrm{j} 2 Y_{\mathrm{TE}_{11}}\left(\omega_{i}\right) \frac{1-S_{11}^{e}\left(\omega_{i}\right)}{1+S_{11}^{e}\left(\omega_{i}\right)}, \quad i=1,2,3 .
\end{aligned}
$$

The value of $L_{o}^{\mathrm{eq}}$ can finally be computed from the following expression:

$$
\frac{1}{\omega_{i}}\left(L_{o}^{\mathrm{eq}}\right)^{-1}=\omega_{i} C_{0}+\frac{\omega_{i}}{\sqrt{\omega_{c}^{2}-\omega_{i}^{2}}} A+\mathrm{j} 2 Y_{\mathrm{TE}_{11}}\left(\omega_{i}\right) \frac{1-S_{11}^{o}\left(\omega_{i}\right)}{1+S_{11}^{o}\left(\omega_{i}\right)}
$$

where $\omega_{i}$ can be one of the frequencies employed in the previous equation.

\section{ACKNOWLEDGMENT}

Author F. Medina would like to thank Prof. Y. Hao, Queen Mary University of London (QMUL), London, U.K., for his continuous support and fruitful discussions.

\section{REFERENCES}

[1] T. W. Ebbesen, H. J. Lezec, H. F. Ghaemi, T. Thio, and P. A. Wolff, "Extraordinary optical transmission through sub-wavelength hole arrays," Nature, vol. 391, pp. 667-669, Feb. 1998.

[2] C. Genet and T. W. Ebbesen, "Light in tiny holes," Nature, vol. 445, pp. 39-46, Jan. 2007.

[3] F. J. García-de-Abajo, "Colloquium: Light scattering by particle and hole arrays," Rev. Modern Phys., vol. 79, pp. 1267-1290, Oct.-Dec. 2007.
[4] F. J. García-Vidal, L. Martín-Moreno, T. W. Ebbesen, and L. Kuipers, "Light passing through subwavelength apertures," Rev. Modern Phys., vol. 82, pp. 729-787, Jan.-Mar. 2010.

[5] L. Martin-Moreno, F. J. Garcia-Vidal, H. J. Lezec, K. M. Pellerin, T. Thio, J. B. Pendry, and T. W. Ebbesen, "Theory of extraordinary optical transmission through subwavelength hole arrays," Phys. Rev. Lett., vol. 86, pp. 1114-1117, Feb. 2001

[6] M. Beruete, M. Sorolla, I. Campillo, J. S. Dolado, L. Martín-Moreno, J. Bravo-Abad, and F. J. García-Vidal, "Enhanced millimeter-wave transmission through subwavelength hole arrays," Opt. Lett., vol. 29, no. 21, pp. 2500-2502, Nov. 2004.

[7] M. Beruete, M. Sorolla, I. Campillo, J. S. Dolado, L. Martín-Moreno, J. Bravo-Abad, and F. J. García-Vidal, "Enhanced millimeter-wave transmission through quasioptical subwavelength perforated plates," IEEE Trans. Antennas Propag., vol. 53, no. 6, pp. 1897-1903, Jun. 2005.

[8] X.-Y. He, X.-N. Fu, and Y.-W. Luo, "Analysis of the extraordinary transmission properties of arrays of subwavelength holes on a metal film in the terahertz region," J. Mod. Opt., vol. 56, no. 15, pp. 1698-1703, Sep. 2009.

[9] S. A. Kuznetsov, M. Navarro-Cía, V. V. Kubarev, A. V. Gelfand, M. Beruete, I. Campillo, and M. Sorolla, "Regular and anomalous extraordinary optical transmission at the THz-gap," Opt. Exp., vol. 17, no. 14, pp. 11730-11738, Jul. 2009.

[10] H. J. Lezec, A. Degiron, E. Devaux, R. A. Linke, L. Martín-Moreno, F. J. García-Vidal, and T. W. Ebbesen, "Beaming light from a subwavelength aperture," Science, vol. 297, pp. 820-822, 2002.

[11] A. A. Oliner and D. R. Jackson, "Leaky surface-plasmon theory for dramatically enhanced transmission through a subwavelength aperture, Part I: Basic features," in IEEE AP-S Int. Symp., Jun. 22-27, 2003, vol. 2, pp. 1091-1094.

[12] D. R. Jackson, T. Zhao, J. T. Williams, and A. A. Oliner, "Leaky surface-plasmon theory for dramatically enhanced transmission through a sub-wavelength aperture, Part II: Leaky-wave antenna model," in IEEE AP-S Int. Symp., Jun. 22-27, 2003, vol. 2, pp. 1095-1098.

[13] S. S. Akarca-Biyikli, I. Bulu, and E. Ozbay, "Enhanced transmission of microwave radiation in one-dimensional metallic gratings with subwavelength aperture," App. Phys. Lett., vol. 85, no. 7, pp. 1098-2000, Aug. 2004.

[14] V. G. Sologub, V. P. Schestopalov, and G. G. Polovnikov, "Diffraction of electromagnetic waves on the grating with narrow slits," Russian $J$. Tech. Phys., vol. 37, no. 4, pp. 667-679, 1967.

[15] J. A. Porto, F. J. García-Vidal, and J. B. Pendry, "Transmission resonances on metallic gratings with very narrow slits," Phys. Rev. Lett., vol. 83, no. 14, pp. 2845-2848, Oct. 1999.

[16] H. J. Rance, O. K. Hamilton, J. R. Sambles, and A. P. Hibbins, "Phase resonances on metal gratings of identical, equally spaced alternately tapered slits," App. Phys. Lett., vol. 95, pp. 041905(1)-041905(3), Oct. 2009.

[17] J. Bravo-Abad, A. Degiron, F. Przybilla, C. Genet, F. J. Garciía-Vidal, L. Martín-Moreno, and T. W. Ebbesen, "How light emerges from an illuminated array of subwavelength holes," Nature Phys., vol. 2, pp. 120-123, Feb. 2006.

[18] J. B. Pendry, L. Martín-Moreno, and F. J. García-Vidal, "Mimicking surface plasmons with structured surfaces," Science, vol. 305, pp. 847-848, Aug. 2004.

[19] F. J. García-de-Abajo and J. J. Sáenz, "Electromagnetic surface modes in structured perfect-conductor surfaces," Phys. Rev. Lett., vol. 95, pp. 233901-1-233901-4, Dec. 2005.

[20] W. L. Barnes, A. Dereux, and T. W. Ebbesen, "Surface plasmon subwavelength optics," Nature, vol. 424, pp. 824-830, Aug. 2003.

[21] C. R. Williams, S. R. Andrews, S. A. Maier, A. I. FernándezDomínguez, L. Martín-Moreno, and F. J. García-Vidal, "Highly confined guiding of terahertz surface plasmon polaritons on structured metal surfaces," Nature Photonics, vol. 2, pp. 175-179, Mar. 2008.

[22] M. J. Lockyear, A. P. Hibbins, and J. R. Sambles, "Microwave surfaceplasmon-like modes on thin metamaterials," Phys. Rev. Lett., vol. 102, pp. 073901-1-073901-4, Feb. 2009.

[23] E. Moreno, L. Martín-Moreno, and F. J. García-Vidal, "Extraordinary optical transmission without plasmons: The $s$-polarization case," $J$ Opt. A, Pure Appl. Opt., vol. 8, pp. S94-S97, 2006, 10.1088/14644258/8/4/S07.

[24] N. G. Don and A. A. Kirilenko, "Eigenoscillations of plane junctions and total transmission through small holes," in Proc. 37th Eur. Microw. Conf., Munich, Germany, Oct. 8-12, 2007, pp. 24-27.

[25] F. Medina, F. Mesa, and R. Marqués, "Equivalent circuit model to explain extraordinary transmission," in IEEE MTT-S Int. Microw. Symp. Dig., Atlanta, GA, Jun. 15-20, 2008, pp. 213-216. 
[26] F. Medina, F. Mesa, and R. Marqués, "Extraordinary transmission through arrays of electrically small holes from a circuit theory perspective," IEEE Trans. Microw. Theory Tech., vol. 56, no. 12, pp. 3108-3120, Dec. 2008.

[27] R. Gordon, "Bethe's aperture theory for arrays," Phys. Rev. A, Gen. Phys., vol. 76, pp. 053806(1)-053806(5), 2007.

[28] Y. Pang, A. N. Hone, P. P. M. So, and R. Gordon, "Total optical transmission through a small hole in a metal waveguide screen Bethe's aperture," Opt. Exp., vol. 17, no. 6, pp. 4433-4441, 2009.

[29] N. G. Don, A. A. Kirilenko, and S. L. Senkevich, "New type of eigenoscillations and total-transmission resonance through an iris with below-cutoff hole in a rectangular waveguide," Radiophys. Quantum Electron., vol. 51, no. 2, pp. 101-108, Feb. 2008.

[30] F. Medina, J. A. Ruiz-Cruz, F. Mesa, J. Rebollar, J. R. Montejo-Garai, and R. Marqués, "Experimental verification of extraordinary transmission without surface plasmons," Appl. Phys. Lett., vol. 95, pp. 0711021-071102-3, Aug. 2009.

[31] A. A. Kirilenko and A. O. Perov, "On the common nature of the enhanced and resonance transmission through the periodical set of holes," IEEE Trans. Antennas Propag., vol. 56, no. 10, pp. 3210-3216, Oct. 2008.

[32] A. G. Schuchinsky, D. E. Zelenchuk, and A. M. Lerer, "Enhanced transmission in microwave arrays of periodic sub-wavelength apertures," $J$. Opt. A, Pure Appl. Opt., vol. 7, pp. S102-S109, 2005.

[33] F. Medina, F. Mesa, and D. C. Skigin, "Extraordinary transmission through arrays of slits: A circuit theory model," IEEE Trans. Microw. Theory Tech., vol. 58, no. 1, pp. 105-115, Jan. 2010.

[34] K. Y. Bliokh, Y. P. Bliokh, V. Freilikher, S. Savel'ev, and F. Nori, "Colloquium: Unusual resonators: Plasmonics, metamaterials, and random media," Rev. Mod. Phys., vol. 80, pp. 1201-1213, Oct.-Dec. 2008.

[35] R. H. MacPhie and K. L. Wu, "Scattering at the junction of a rectangular waveguide and a larger circular waveguide," IEEE Trans. Microw. Theory Tech., vol. 43, no. 9, pp. 2041-2045, Sep. 1995.

[36] K.-L. Chan and S. Judah, "Two port scattering at an elliptical-waveguide junction," IEEE Trans. Microw. Theory Tech., vol. 45, no. 8, pp. 1255-1262, Aug. 1997.

[37] N. Marcuvitz, Waveguide Handbook, ser. MIT Radiat. Lab. New York: McGraw-Hill, 1951, vol. 10, new ed., IEE/Peregrinus, 1986.

[38] R. E. Collin, Field Theory of Guided Waves, 2nd ed. Oxford, U.K.: Oxford Univ. Press/IEEE Press, 1995.

[39] L. Martín-Moreno and F. J. García-Vidal, "Optical transmission through circular hole arrays in optically thick metal films," Opt. Exp., vol. 12, no. 16, pp. 3619-3628, Aug. 2004.

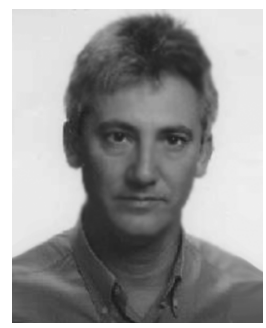

Francisco Medina (M'90-SM'01-F'10) was born in Puerto Real, Cádiz, Spain, in November 1960. He received the Licenciado and Doctor degrees from the University of Seville, Seville, Spain, in 1983 and 1987 respectively, both in physics.

From 1986 to 1987, he spent the academic year with the Laboratoire de Microondes de l'ENSEEIHT, Toulouse, France. From 1985 to 1989, he was an Assistant Professor with the Department of Electronics and Electromagnetism, University of Seville, where, since 1990, he has been an Associate Professor of electromagnetism. He is currently a Full Professor of electromagnetism (since July 2009) and Head of the Microwaves Group. He is a Reviewer of over 30 IEE, IEEE, and American Physics Society journals. His research interest includes analytical and numerical methods for guiding, resonant, and radiating structures, passive planar circuits, periodic structures, and the influence of anisotropic materials (including microwave ferrites) on such systems. He is also interested in artificial media modeling and design and extraordinary transmission phenomena.

Dr. Medina is a Fellow of the Massachusetts Institute of Technology (MIT) Electromagnetics Academy. He is on the Editorial Board of the International Journal of RF and Microwave Computer-Aided Engineering. He is a reviewer of the IEEE TRANSACTIONS ON MicRowaVE THEORY AND TECHNIQUES He has been a member of the Technical Programme Committees (TPCs) of several international and local conferences and has organized a few conferences and workshops. He was the recipient of a Spanish Ministerio de Educación y Ciencia (MEC) Research Scholarship and a French Ministére de la Recherche et la Technologie Scholarship.

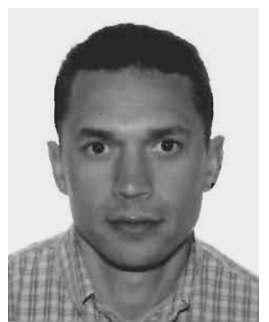

Francisco Mesa (M'93) was born in Cádiz, Spain, in April 1965. He received the Licenciado and Doctor degrees in physics from the Universidad de Sevilla, Seville, Spain, in 1989 and 1991, respectively.

$\mathrm{He}$ is currently an Associate Professor with the Departamento de Física Aplicada 1, Universidad de Sevilla, Seville, Spain. His research interests focus on electromagnetic propagation/radiation in planar structures. He is also involved with extraordinary transmission and metamaterials.

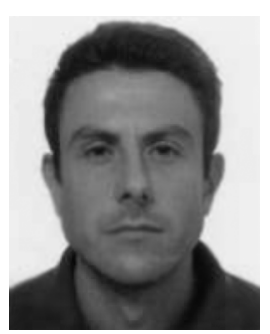

Jorge A. Ruíz-Cruz received the Ingeniero de Telecomunicación degree and Ph.D. degree from the Universidad Politécnica de Madrid (UPM), Madrid, Spain, in 1999 and 2005, respectively.

Since 1999, he has collaborated with the Grupo de Electromagnetismo Aplicado y Microondas, UPM. Prior to beginning the doctoral program, he was with RYMSA in 2000. Since 2006, he has been with the Universidad Autónoma de Madrid, Madrid, Spain. His current research interests are the computer-aided design of microwave passive devices and circuits (filters, multiplexers, ortho-modes, etc.).

Dr. Ruíz-Cruz was the recipient of an award of Telefónica Foundation-COIT for his doctoral thesis and an award from the UPM.

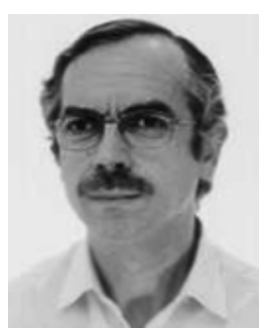

Jesús M. Rebollar was born in Beasain (Gipuzkoa), Spain, in 1953. He received the Ingeniero de Telecomunicación degree and Doctor degree from the Universidad Politécnica de Madrid, Madrid, Spain, in 1975 and 1980, respectively.

Since 1976, he has been with the Grupo de Electromagnetismo Aplicado y Microondas, Universidad Politécnica de Madrid, as an Assistant Professor until 1982, and then as an Associate Professor until 1988, when he was appointed Professor of Teoría Electromagnética. His research interest include electromagnetic wave propagation in waveguide structures, interactions of electromagnetic fields with biological tissues, and particularly computer-aided design (CAD) for microwave and millimeter-wave passive devices: filters, multiplexers, polarizers, orthomode transducers, beam forming networks, etc. He has designed many of the above components for communication systems on board satellites.

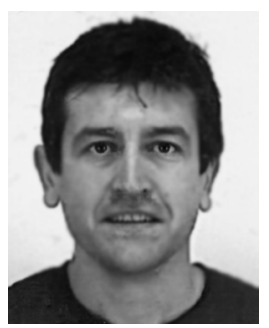

José R. Montejo-Garai was born in Vitoria-Gasteiz, Spain, in 1965. He received the Ingeniero de Telecomunicación and Ph.D. degrees from the Universidad Politécnica de Madrid, Madrid, Spain, in 1990 and 1994, respectively.

Since 1989, he has been with the Grupo de Electromagnetismo Aplicado y Microondas, Universidad Politécnica de Madrid, as an Assistant Professor until 1996, after which he became an Associate Professor. His research interests include the analysis and characterization of waveguide structures, advanced synthesis theory, and computer-aided design (CAD) for microwave and millimeter-wave passive devices: filters, multiplexers, orthomode transducers, beam-forming networks, etc. He has designed numerous passive microwave devices for communication satellites. 